\section{TREC: A tool kit for programming cognitive experiments in Applesoft BASIC}

\author{
JAIME SANMARTIN \\ Universitat de València, València, Spain
}

The development of microcomputer programs for experimental applications presents two main problems. First, it is usually necessary to implement a timer for measurement of response latencies and stimulus exposure duration (Dlhopolsky, 1983b). Second, programming in BASIC is usually inadequate, because it is difficult to synchronize the program with the videoscan of the CRT and to measure the time (Dlhopolsky, 1983a).

The package described in this paper has been developed with the purpose of facilitating the interface between the routines and the user-programmer. Quick and versatile programming in Applesoft BASIC of varied routines for cognitive psychology experimentation is possible by accessing binary routines to control stimulus exposure, generate time delays, and measure response latency.

This package offers more possibilities than do others (e.g., Algarabel, 1983; Poffel \& Gregory, 1984). Its main virtues are versatility and ease of use; it allows people with few programming skills to implement a wide range of experimental designs, such as free recall, recognition memory, and decision. It also is possible to program a tachistoscope, adapting ideas discussed by others (e.g., Diener \& Smee, 1984; Dlhopolsky, 1983b; Femano \& Pfaff, 1983). A subsidiary application would be to let students interact with basic experimental problems in laboratory settings by writing their own programs.

\section{Requirements}

The program runs on an Apple Ile with a disk unit. It cannot run adequately on an Apple $\mathrm{II}, \mathrm{II}$, or Ilc, because, without hardware and software modifications, it is not possible to have access to vertical blanking synchronization. For more details, see the technical reference manuals and other papers (Apple Computer, Inc., 1982; Diener \& Smee, 1984).

\section{General Description}

The program in binary has a length of 517 (\$205) bytes, and it begins at address $34048(\$ 8500)$. When the BRUN TREC.OBJ instruction is executed, the first part of the program sets the HIMEM and allows the various functions to be accessed by using the " $\&$ " prefix.

A mini-interpreter, which is included in the second part of the program, evaluates the specified functions and transfers the variable content (pointers).

Address correspondence to Jaime Sanmartin, Departament de Psicologia Experimental, Facultat de Psicologia, Av. Blasco Ibañez 21, 46010 Valencia, Spain.
In the third part, the routines that execute the different functions are included. There are two basic routines to control time: a delay routine and a timer routine. The timer routine stops and returns to the main program when a key is pressed, or when a 2-byte value in milliseconds $(65,535 \mathrm{msec})$ is exceeded. Time values are specified in milliseconds, with the range allowed by an integer variable ( 2 bytes). The precision, although it depends on the configuration of the BASIC program, is adequate for verbal learning and lexical decision experiments. The software clock has been calibrated in both Apple IIe American series and Apple Ie European series. A third BASIC routine synchronizes the stimulus displays with vertical blanking, for the upper-left corner of the monitor, testing the address 49177 (\$CO19) (Apple Computer, Inc., 1982; Diener \& Smee, 1984). Stimulus length is limited to one line.

The complete program is developed in a way that permits new functions that use or combine the existing routines to be added without problems.

\section{Utilities and Functions}

The utilities are accessible by typing BASIC commands in a function-like way, using the " $\&$ " prefix and a set of arguments within parentheses:

$$
\text { \& NAME (argument1, argument2, ...) }
$$

The different names are formed with a reserved word of Applesoft BASIC to simplify its codification in tokens. The arguments must be integer or string variables (not real variables, numbers, strings, or direct expressions). These variables are the input-output media of the function. The program interpreter gives a syntax error (number 16) if the name is not specified correctly, and it gives an illegal error (number 163) if the argument is not an appropriate variable type.

The following functions are available:

\& DEL (D\%). Produces a time delay of as many milliseconds as the ones specified by the variable $\mathrm{D} \%$.

\& WAIT (L\%, R\%). Measures the time from execution of the command until a key is pressed. Response latency (in milliseconds) is available in L\% and the ASCII code of the pressed key in $\mathrm{R} \%$ (number 207 for openapple and 211 for solid-apple.

\& EXP DEL (W\$, D\%). Displays the string stored in $\mathrm{W} \$$, in the last position used (e.g., with VTAB and HTAB) for the time stored in D\%. The string is then replaced with the character stored in address $\$ 1 \mathrm{E}$. Both processes are synchronized with the vertical blanking.

\& EXP WAIT (W\$, L\%, R\%). The string stored in W\$ is displayed in the same way as \& EXP DEL, but the display persists until a key is pressed. Response latency is returned in L\% and the key code in $\mathrm{R} \%$, as in \& WAIT (L\%, R\%). 
\& EXP DEF (W\$, D\%, L\%, R\%). Displays the string in W\$ for D\% milliseconds, as in \& EXP DEL. Response latency is returned in $\mathrm{L} \%$ and the key code in $\mathrm{R} \%$, as in \& WAIT ( $\mathrm{L} \%, \mathrm{R} \%)$.

\section{Program Availability}

The binary (assembled) program and its text source with programming notes are available on disk by sending $\$ 10$ to the author.

\section{REFERENCES}

Algarabel, S. (1983). MEMORIA: A computer program for experimental control of verbal learning and memory experiments with the Apple II microcomputer. Behavior Research Methods \& Instrumentation, 15, 394.

APPLE COMPUTER, INC. (1982). Apple II reference manual . Cupertino, CA: Author.
DIENER, D., \& SMEE, W. P. (1984). Apple tachistoscope. Behavior Research Methods, Instruments, \& Computers, 16, 540-544.

DLHopolsky, J. G. (1983a). Limitations of high-level microcomputer languages in software designed for psychological experimentation. Behavior Research Methods \& Instrumentation, 15, 459-464.

DLhopolsKy, J. G. (1983b). Machine language millisecond timer for the Z-80 microprocessor. Behavior Research Methods \& Instrumentation, 15, 511-520.

Femano, P. A., \& Pfaff, D. W. (1983). Time-interval acquisition on 6502-based microcomputer. Behavior Research Methods \& Instrumentation, 15, 521-529.

PoFfel, S. A., \& GRegory, R. J. (1984). START: Stimulus and response tools for experiments in memory, learning, cognition, and perception. Behavior Research Methods, Instruments, \& Computers, 16, 409-410.

(Revision accepted for publication July 24, 1987.)

\section{NOTICE}

\section{Nominations for the Editorship of Memory \& Cognition}

Nominations are solicited for the editorship of Memory \& Cognition. The term of the present editor, Alice F. Healy, expires at the end of 1989 . The new editor will begin to receive manuscripts in 1989, with an official 4-year term to begin January 1, 1990. Nominations must be submitted by March 1, 1988.

Nominations (including self-nominations) should be sent to:

Richard M. Shiffrin

Chair, Memory \& Cognition Search Committee

Department of Psychology

Indiana University

Bloomington, IN 47405 